\title{
Direct observation of apical oxygen vacancies in the high-temperature superconductor $\mathrm{YBa}_{2} \mathrm{Cu}_{3} \mathrm{O}_{7-x}$
}

\author{
Steven T. Hartman $\odot,{ }^{1,{ }^{*}}$ Bernat Mundet, ${ }^{2,{ }^{*}}$ Juan-Carlos Idrobo, ${ }^{3}$ Xavier Obradors, ${ }^{2}$ Teresa Puig, ${ }^{2}$ \\ Jaume Gázquez $\odot,{ }^{2, \dagger}$ and Rohan Mishra $\oplus^{4,1, \$}$ \\ ${ }^{1}$ Institute of Materials Science and Engineering, Washington University in St. Louis, St. Louis, Missouri 63130, USA \\ ${ }^{2}$ Institut de Ciéncia de Materials de Barcelona (ICMAB-CSIC), Campus UAB, Bellaterra, 08193 Barcelona, Spain \\ ${ }^{3}$ Center for Nanophase Materials Sciences, Oak Ridge National Laboratory, Oak Ridge, Tennessee 37831, USA \\ ${ }^{4}$ Department of Mechanical Engineering and Materials Science, Washington University in St. Louis, St. Louis, Missouri 63130, USA
}

(Received 3 April 2019; revised manuscript received 27 September 2019; published 22 November 2019)

\begin{abstract}
The properties of the high-temperature superconductor $\mathrm{YBa}_{2} \mathrm{Cu}_{3} \mathrm{O}_{7-x}$ (YBCO) depend on the concentration of oxygen vacancies $\left(V_{\mathrm{O}}\right)$. It is generally agreed upon that $V_{\mathrm{O}}$ form in the $\mathrm{CuO}$ chains, even at low concentrations where the critical temperature for superconductivity peaks $(x \approx 0.07)$, with only a handful of reports suggesting the presence of $V_{\mathrm{O}}$ at the apical sites. In this paper, we show direct evidence of apical $V_{\mathrm{O}}$ in optimally doped YBCO samples. Using density-functional-theory calculations, we predict that isolated $V_{O}$ are equally favorable to form in either the $\mathrm{CuO}$ chains or the apical sites, which we confirm using atomic-resolution scanning transmission electron microscope imaging and spectroscopy. We further show that apical $V_{\mathrm{O}}$ lead to significant lattice distortions and changes in the electronic structure of YBCO, indicating they should be considered on an equal footing with chain $V_{\mathrm{O}}$ to understand the superconducting properties of $\mathrm{YBCO}$ in the optimal doping region.
\end{abstract}

DOI: 10.1103/PhysRevMaterials.3.114806

\section{INTRODUCTION}

Subtle changes in structure and stoichiometry stemming from defects influence many functional properties of ceramics. A paradigmatic example is the high-temperature superconductor $\mathrm{YBa}_{2} \mathrm{Cu}_{3} \mathrm{O}_{7-x}$ (YBCO), where its critical temperature $\left(T_{\mathrm{c}}\right)$ and critical current density $\left(J_{\mathrm{c}}\right)$ are governed by oxygen vacancies $\left(V_{\mathrm{O}}\right)$ [1-3]. The structure of YBCO, shown in Fig. 1(a), is that of an oxygen-deficient perovskite. Oxygen is absent from the $\mathrm{Y}$ layers, and from half of the sites in the $\mathrm{CuO}$ layers present between two $\mathrm{BaO}$ layers, which are commonly referred to as $\mathrm{CuO}$ chains [4]. The bridging oxygen in these chains can also be removed under reducing conditions, allowing the stoichiometry to vary continuously from $\mathrm{YBa}_{2} \mathrm{Cu}_{3} \mathrm{O}_{7}$ to $\mathrm{YBa}_{2} \mathrm{Cu}_{3} \mathrm{O}_{6}$. Chain $V_{\mathrm{O}}$ leads to structural distortions in their vicinity and dopes the system with electrons [1]. Optimal doping, at $x=0.07$, gives a maximum $T_{\mathrm{c}}$ of $92 \mathrm{~K}$, while $x>0.55$, destroys the superconducting state [1], thus controlling the oxygen stoichiometry allows a way to tune $T_{\mathrm{c}} \cdot J_{\mathrm{C}}$ is also modulated by the oxygen content, since the magnetic vortex pinning is sensitive to both the carrier concentration and the distortions around $V_{\mathrm{O}}[2,3,5]$.

Oxygen vacancies have received enormous attention, but nearly all past studies have focused on the chain $V_{\mathrm{O}}, \mathrm{O}(1)$ in Fig. 1(a). There are three other oxygen sites in YBCO, one apical site in the $\mathrm{BaO}$ layer, $\mathrm{O}(4)$, and two inequivalent sites in the $\mathrm{CuO}_{2}$ planes, $\mathrm{O}(2)$ and $\mathrm{O}(3)$. The attention to the chain vacancies over others can be attributed to two factors. First,

\footnotetext{
*These authors contributed equally to this work.

${ }^{\dagger}$ Corresponding authors: jgazqueza@ gmail.com

‡Corresponding authors: rmishra@wustl.edu
}

the full deoxygenation of the chains under reducing conditions suggests that the chain vacancy ought to be energetically preferable. Second, the position of $V_{\mathrm{O}}$ is commonly detected using diffraction-based techniques involving either $\mathrm{x}$ rays, neutrons, or electrons. However, owing to their large interaction volume, such techniques are sensitive only when the vacancy concentration is large; and at large concentrations, $V_{\mathrm{O}}$ in YBCO prefers to order in the chains [6]. Both of these factors obscure the behavior of $V_{\mathrm{O}}$ at small concentrations, or low $x$, where $T_{\mathrm{c}}$ peaks. Nonetheless, there are a few reports of $V_{\mathrm{O}}$ at sites other than the chains. For instance, neutron diffraction studies [7-11] have indicated small vacancy concentrations at the apical site $\mathrm{O}(4)$, depending on the annealing process. There have been attempts at atomic-scale characterization of $V_{\mathrm{O}}$ on surfaces of cleaved YBCO crystals having optimal doping using scanning tunneling microscopy (STM) [12-15]. Pan et al. [12] observed modulations in the intensity of the $\mathrm{BaO}$ surface and attributed them to apical vacancies; however, subsequent STM studies did not find any apical vacancies [14] and concluded that the previously observed modulations were due to electronic ordering in the underlying $\mathrm{CuO}_{2}$ plane [13]. To summarize, while there is some evidence that apical vacancies can form in YBCO at optimal doping, the results are inconclusive owing to either the low spatial resolution of the characterization techniques or to the sample preparation methods. Furthermore, little is known about the conditions that favor the formation of apical vacancies and their effect on YBCO's structure and properties.

In this paper, we provide direct evidence of apical $V_{\mathrm{O}}$ in optimally doped thin films and single crystals of YBCO by using a combination of aberration-corrected scanning transmission electron microscopy (STEM) and first-principles density-functional-theory (DFT) calculations. Based on DFT 
(a)

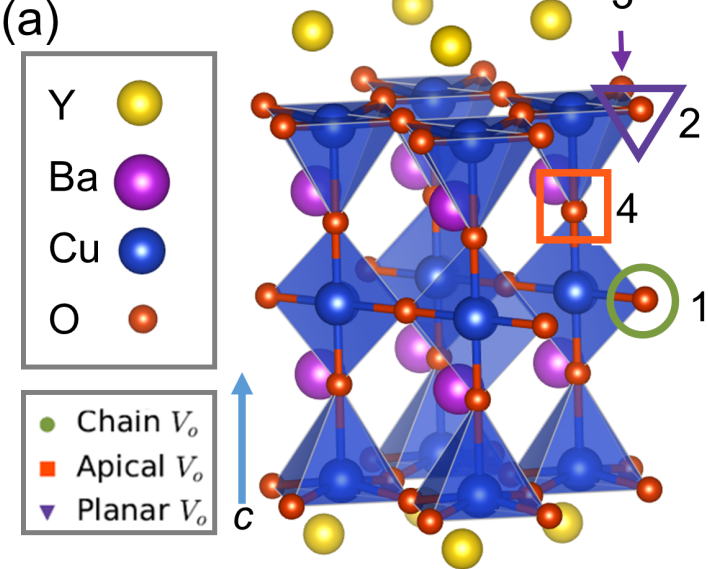

(b)

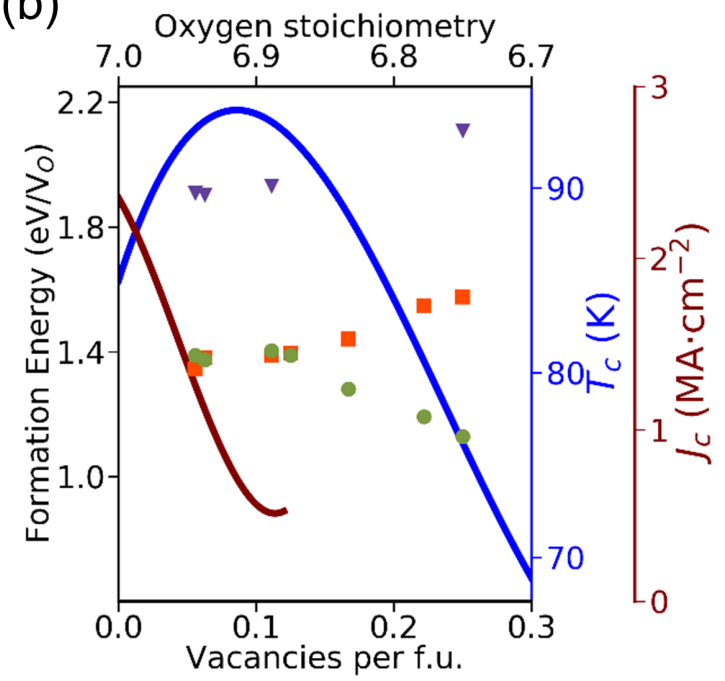

FIG. 1. (a) The atomic structure of fully oxygenated $\mathrm{YBa}_{2} \mathrm{Cu}_{3} \mathrm{O}_{7}$ $\left(\mathrm{YBCO}_{7}\right)$. The locations of the four possible oxygen vacancies are marked. (b) Oxygen vacancy formation energy as a function of oxygen stoichiometry, using the same colors and symbols as (a). The solid blue line shows $T_{\mathrm{c}}$, based on experimental data (Ref. [17], while the solid brown line shows $J_{\mathrm{C}}$ (Ref. [2]. $\mathrm{O}(2)$ and $\mathrm{O}(3)$ are combined because they have nearly identical formation energies.

calculations, we find that isolated apical $V_{O}$ have a small formation energy, comparable to that of isolated chain $V_{\mathrm{O}}$, and should exist across the entire doping range. Using atomicresolution STEM imaging, we show that apical vacancies are most common in the vicinity of $\mathrm{YBa}_{2} \mathrm{Cu}_{4} \mathrm{O}_{8}$ (YBCO-124) intergrowths, which are prevalent stacking faults formed by the inclusion of a second layer of $\mathrm{CuO}$ chains. Furthermore, the observed distortions around the apical vacancies in the STEM images match the equivalent DFT-optimized structural distortions, confirming their origin. We find that the apical vacancies modify the electronic structure of adjacent plane $\mathrm{Cu}$ atoms, as shown by simultaneously acquired electron energy loss spectra (EELS) in STEM. DFT calculations show that the apical vacancies increase the electron density of the adjacent superconducting $\mathrm{CuO}_{2}$ planes. Finally, we compare our results with prevalent models of cuprate superconductivity, and consider how apical vacancies might affect YBCO's superconductive properties.

\section{RESULTS AND DISCUSSION}

To assess the favorability of different $V_{\mathrm{O}}$ in the optimal doping region, we calculated the formation energies $\left(E_{\text {form }}\right)$ of apical, chain, and planar $V_{\mathrm{O}}$ in YBCO by varying $x$ from 0.05 to 0.25 vacancies per formula unit. We only considered one vacancy type when varying the concentration; interaction of different vacancy types is briefly addressed in the Supplemental Material [16]. For $x<0.15$, we find that isolated apical $\mathrm{O}(4)$ and chain $\mathrm{O}(1)$ vacancies have similar $E_{\text {form }}$, as shown in Fig. 1(b). For instance, at $x=0.06, E_{\text {form }}$ of an isolated apical and chain $V_{\mathrm{O}}$ is 1.35 and $1.39 \mathrm{eV}$, respectively, suggesting that the two are likely to exist at similar concentrations. Although a previous work has examined the ordering of chain vacancies in YBCO at higher $V_{O}$ concentrations using DFT [6], we are not aware of any first-principles studies comparing the stability of isolated apical and chain vacancies. It is not favorable to form vacancies at the planar sites $\mathrm{O}(2)$ and $\mathrm{O}(3)$, as they have $E_{\text {form }}$ of 1.90 and $1.91 \mathrm{eV}$, respectively, at $x=0.06$. At higher concentrations, we find that chain vacancy formation benefits from ordering. For instance, for $x=0.25, E_{\text {form }}$ of the chain vacancy decreases to $1.13 \mathrm{eV}$, while it increases for the apical vacancy to $1.58 \mathrm{eV}$. We find that the lowest energy ordering of chain $V_{\mathrm{O}}$ is an entire chain emptied of oxygen, in agreement with previous experiments [4] and theory [6]. Additional results on $V_{\mathrm{O}}$ ordering and formation energy of apical $V_{\mathrm{O}}$ in other cuprates are included in the Supplemental Material.

We have used STEM annular bright-field (ABF) imaging, which is sensitive to lighter elements [18,19], to identify oxygen vacancies in YBCO samples synthesized by chemical solution deposition; the synthesis and characterization procedures are described in the Supplemental Material. Figure 2(a) shows an ABF image of YBCO. Figure 2(b) shows the same image with its contrast inverted to show the oxygen columns in the $\mathrm{BaO}$ layers and the $\mathrm{CuO}$ chains with more clarity. We show the intensity trace measured along two $\mathrm{BaO}$ layers close to a YBCO-124 stacking fault. While the Ba columns have the same intensity for both layers, the $\mathrm{O}(4)$ column closer to the film surface has a lower intensity than the $\mathrm{O}(4)$ column farther from the surface, indicating the presence of apical vacancies. We observe a similar intensity difference for pairs of $\mathrm{BaO}$ layers several unit cells away from the faults, with the layer closer to the surface consistently having more apical vacancies. Crystallographically, the near and far planes are equivalent, so the asymmetry in vacancy concentration may be due to kinetic factors during the growth process. We have also performed STEM image simulations on DFToptimized structures containing $\mathrm{O}(4)$ vacancies to directly compare with the experimental images. Figure 2(d) shows a simulated contrast-inverted ABF image of a DFT-optimized supercell having $25 \% V_{\mathrm{O}}$ in one of the $\mathrm{BaO}$ planes. We find good agreement between the simulated and experimental intensity profile changes, confirming the presence of apical $V_{\mathrm{O}}$.

Apical vacancies are expected to create structural distortions in their vicinity that serve as additional evidence of their presence. We have quantified these distortions from the $\mathrm{ABF}$ images by locating the different atomic columns using a center-of-mass refinement method (see the Supplemental Material for details). By measuring the change in various lattice spacings around $\mathrm{BaO}$ layers with or without apical $V_{\mathrm{O}}$, 


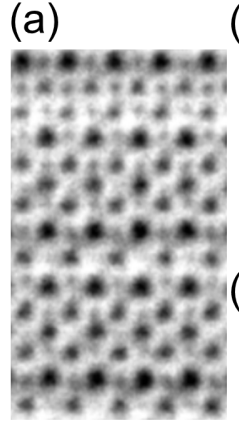

(b)
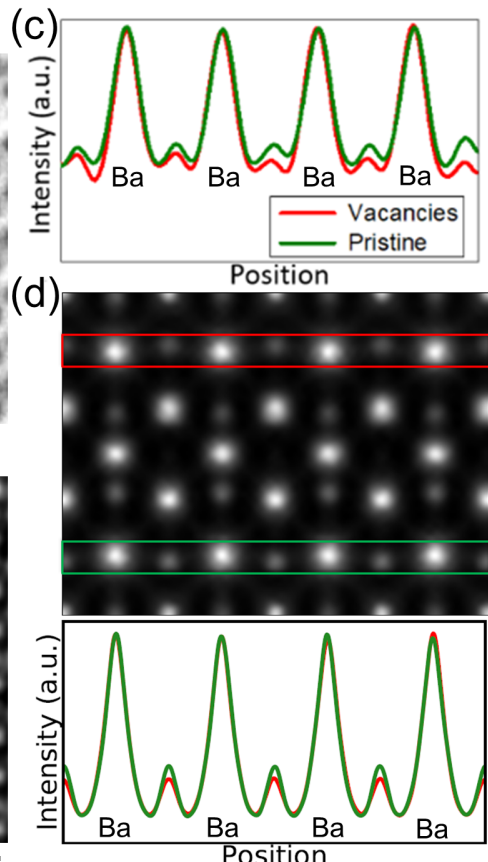

FIG. 2. (a) Raw ABF image of a YBCO thin film with a YBCO124 intergrowth viewed along the [100] zone axis. (b) The same ABF image with its contrast inverted to show the atomic columns as bright spots. The image has been filtered to reduce the noise. Scale bar: $1 \mathrm{~nm}$. (c) Panel showing two horizontal intensity profiles measured along the $\mathrm{BaO}$ planes near to the intergrowth. (d) A simulated contrast-inverted ABF image of a YBCO supercell relaxed with $25 \%$ oxygen vacancies in one of the apical planes, marked with a red box, which corresponds to the red intensity trace.

we have obtained a detailed real-space map of the vacancyinduced structural distortions. For comparison, we have used DFT to optimize a $3 \times 3 \times 1$ supercell of stoichiometric $\mathrm{YBCO}_{7}$ with one apical vacancy. We show the largest distortions caused by the apical $V_{\mathrm{O}}$, both in experiments and DFT calculations, in Fig. 3(a). Due to the apical $V_{\mathrm{O}}$, the spacing along the $c$ axis between $\mathrm{Y}$ and $\mathrm{Ba}$ is reduced by $1.5 \%$ relative to the regions without apical $V_{\mathrm{O}}$. The planar $\mathrm{Cu}$ atom, adjacent to the vacancies, shifts closer to $\mathrm{Y}$, whereas $\mathrm{O}(2)$ and $\mathrm{O}(3)$ atoms shift in the opposite direction, changing the planar $\mathrm{O}-\mathrm{Cu}-\mathrm{O}$ angle from $165 \pm 2^{\circ}$ to $176 \pm 2^{\circ}$. This motion also reduces the interplane $\mathrm{Cu}-\mathrm{Cu}$ distance by $5.3 \%$, and increases the distance between planar $\mathrm{Cu}$ and the remaining apical $\mathrm{O}(4)$ in the atomic column by $8 \pm 3 \%$. We find excellent agreement between these observed distortions and the calculated distortions, as shown in Fig. 3(b). The calculated apical $V_{\mathrm{O}}$ causes the copper atoms above and below to move away by 0.18 and $0.23 \AA$, respectively, flattening the in-plane $\mathrm{O}-\mathrm{Cu}-\mathrm{O}$ bond angle from $163^{\circ}$ to $175^{\circ}$ and buckling the usually straight $\left(180^{\circ}\right)$ chain $\mathrm{O}-\mathrm{Cu}-\mathrm{O}$ angle to $154^{\circ}$. In addition, the adjacent chain oxygen moves towards the vacancy by $0.23 \AA$.

The presence of apical $V_{\mathrm{O}}$ and the associated distortions affect the local electronic structure of YBCO, as observed from simultaneously acquired EEL spectra. Figure 4(a) compares the fine structure of the $\mathrm{O} K$ edge in $\mathrm{CuO}_{2}$ planes and $\mathrm{CuO}$ chains that were acquired from a region of the thin film away from apparent apical $V_{\mathrm{O}}$. Our results match well with (a)

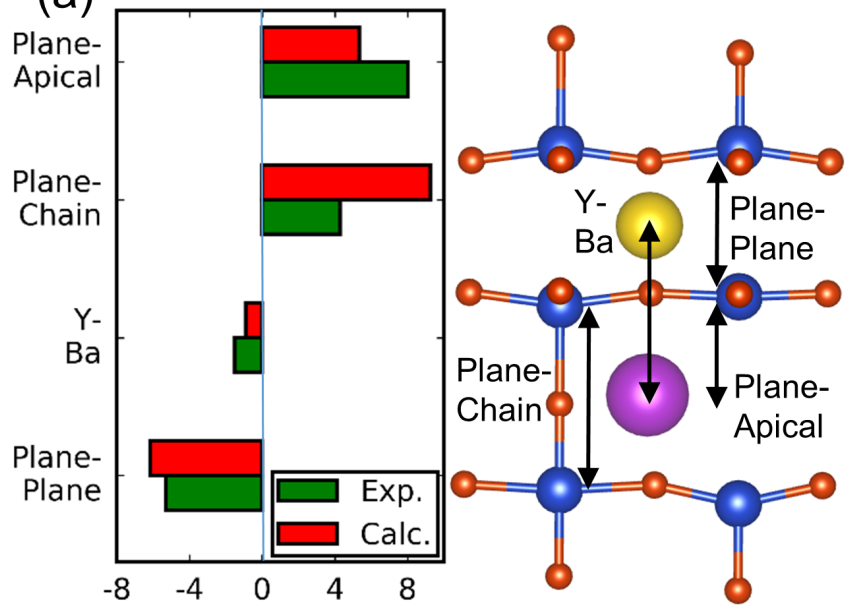

Change in separation (\%)

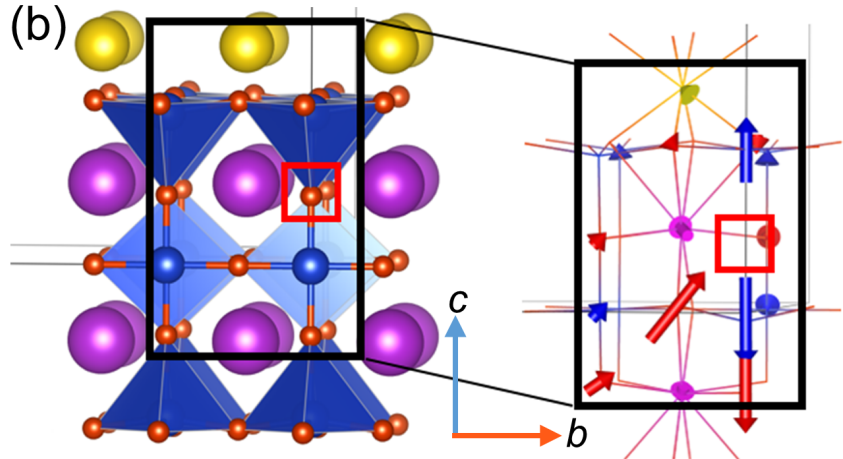

FIG. 3. (a) The change in the interlayer separations for the experimental STEM images and the DFT-calculated YBCO structure with apical vacancies. Each separation is labeled in the schematic. (b) Structural relaxation around an apical $V_{\mathrm{O}}$ as calculated using DFT. The left panel shows the location of the vacancy in the pristine $\mathrm{YBCO}_{7}$, while the right panel is a wireframe of the relaxed structure with vectors showing the atomic displacements. The vectors follow the same color scheme as the atoms; red vectors show $\mathrm{O}$ movement, blue $\mathrm{Cu}$, magenta $\mathrm{Ba}$, and yellow $\mathrm{Y}$. The vector length is magnified fivefold for clarity.

those obtained by Gauquelin et al. [20] from a YBCO single crystal that we take as a reference to validate the quality of our EELS measurements. The prepeak of the $\mathrm{O} K$ edge is sensitive to the filling of the unoccupied, hybridized $\mathrm{O}-2 p$ and $\mathrm{Cu}-3 d$ states, and is observed to shift to higher energies with oxygen vacancies, due to the electron doping [20]. In addition, our observation of the prepeak onset at $527 \mathrm{eV}$ for the $\mathrm{CuO}$ chains and $528.5 \mathrm{eV}$ for the $\mathrm{CuO}_{2}$ planes is consistent with previous results for $\mathrm{YBCO}_{6.97}$ single crystals [20], indicating that our thin films have the optimal oxygen concentration. The EELS edge of $\mathrm{Cu}$ from chains and planes is provided in the Supplemental Material.

We now show the difference in $\mathrm{Cu} L$ and $\mathrm{O} K$ edges in the EEL spectra of two superconducting $\mathrm{CuO}_{2}$ planes with and without adjacent apical $V_{O}$ in Figs. 4(b) and 4(c), respectively. For the planar $\mathrm{O} K$ edge, the most notable difference is the decrease in the intensity of the prepeak in the planes adjacent to the apical $V_{\mathrm{O}}$. We attribute this to electron doping by the adjacent apical $V_{\mathrm{O}}$ of the previously unoccupied $\mathrm{O} 2 p$ states 


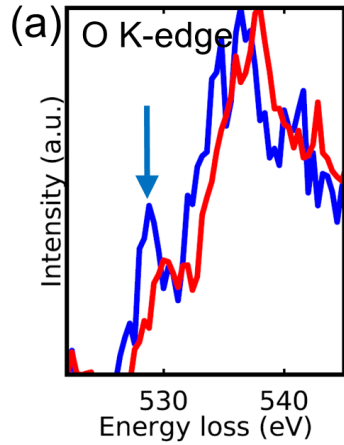

(b)
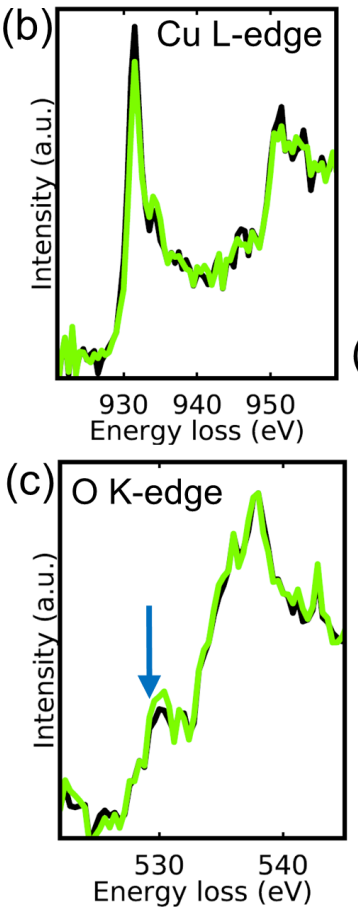

.
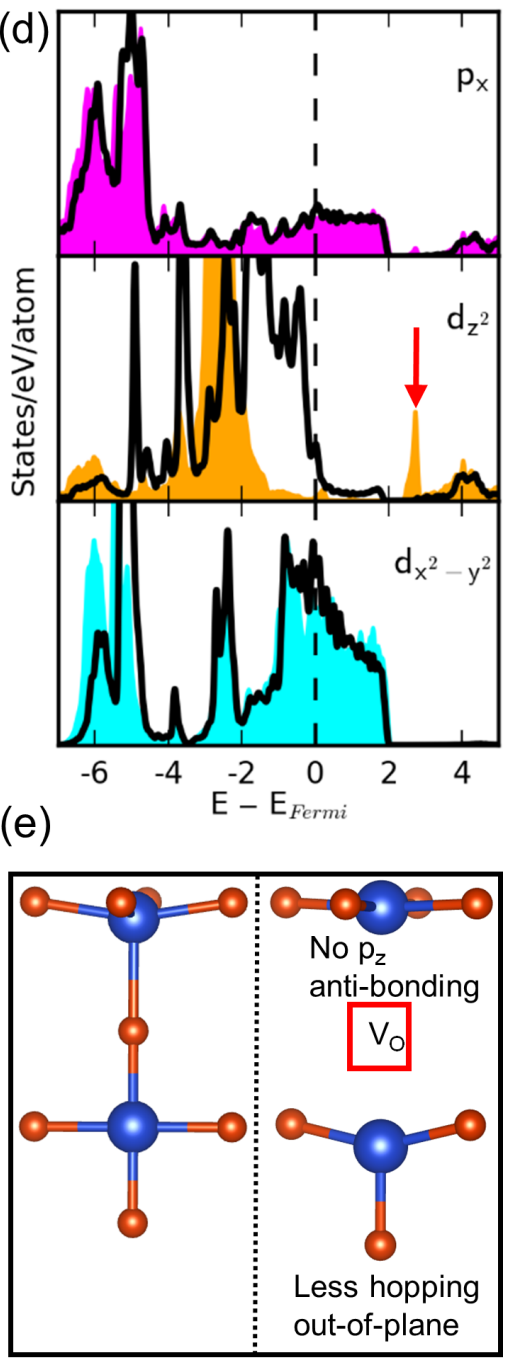

FIG. 4. (a) EELS O $K$-edge spectra obtained from the chain $\mathrm{O}(1)$ (blue) and plane $\mathrm{O}(2), \mathrm{O}(3)$ (red) of optimally doped YBCO thin films. The prepeak is marked with an arrow. (b) Comparison of $\mathrm{Cu}$ $L$-edge spectra from $\mathrm{CuO}_{2}$ planes closer to the film surface (black) with more apical $V_{\mathrm{O}}$ and farther from it with fewer apical $V_{\mathrm{O}}$ (green). (c) Comparison of $\mathrm{O} K$-edge spectra from the same $\mathrm{CuO}_{2}$ planes as in (b). (d) The orbital-projected DOS of the superconducting $\mathrm{CuO}_{2}$ plane atoms directly adjacent to an apical vacancy. The planar $\mathrm{O} p_{x}, \mathrm{Cu} 3 d_{z^{2}}$ and $\mathrm{Cu} 3 d_{x^{2}-y^{2}}$ states are in magenta, gold, and blue, respectively. The states near $3 \mathrm{eV}$, highlighted by an arrow correspond to the axial orbital as proposed by Pavarini et al. [21]. The black line in the DOS plots corresponds to pristine YBCO, shown for comparison. (e) Schematic of the plane-chain system before and after the apical oxygen is removed.

hybridized with $\mathrm{Cu} 3 d$ in the superconducting planes. The $\mathrm{Cu} L$-edge also changes when comparing the two superconducting $\mathrm{CuO}_{2}$ planes. Notice that the intensity of the $\mathrm{Cu} L_{3}$ edge (centered at $931.5 \mathrm{eV}$ ) increases with apical vacancies, which constitutes a second experimental evidence of an electronic reconstruction. The DFT-calculated electronic density of states (DOS) for the planar $\mathrm{Cu}$ and planar $\mathrm{O}$ adjacent to an apical $V_{O}$ are shown in Fig. 4(d), compared with the same atoms in pristine YBCO. The formation of an apical $V_{\mathrm{O}}$ eliminates the overlap between the apical oxygen's $2 p_{z}$ states and the neighboring planar copper's $3 d_{z^{2}}$ states, reducing the bandwidth by $\sim 2 \mathrm{eV}$. This interaction is antibonding near the Fermi energy, so the resulting nonbonding $\mathrm{Cu} 3 d_{z^{2}}$ state drops below the Fermi energy. The remaining planar $\mathrm{Cu}$ DOS at the Fermi energy comes from the $3 d_{x^{2}-y^{2}}$ states, which retain their bandwidth after the apical vacancy formation.

The apical oxygen has been shown to affect the superconducting properties of YBCO. For instance, a recent investigation [22] of the magnetic excitations in cuprates with zero, one, or two apical oxygen per $\mathrm{CuO}_{2}$ plane concluded that the structure without apical oxygen had the most long-range electron hopping. Likewise, any strain or distortion of the $\mathrm{CuO}_{2}$ planes is likely to alter the pair condensation energy and its $J_{\mathrm{C}}$ [3]. Hence, we consider the implications of apical $V_{\mathrm{O}}$.

Pavarini et al., [21] have ascribed an important role to the "axial" molecular orbital, composed of planar $\mathrm{Cu} 4 s$ and $3 d_{z^{2}}$ states antibonding to apical $\mathrm{O} 2 p_{z}$ states. The axial orbital is above the Fermi energy, and its calculated energy is correlated with $T_{\mathrm{c}}$; as the axial orbital approaches the Fermi energy, $T_{\mathrm{c}}$ goes up. Increasing the planar $\mathrm{Cu}-\mathrm{O}(4)$ bond length is predicted to raise $T_{\mathrm{c}}$ by reducing the antibonding contribution to the axial orbital. Removing the apical oxygen is equivalent to a large bond length increase, along with the addition of electrons. It results in the axial orbital, located $\sim 3 \mathrm{eV}$ above Fermi energy as shown in Fig. 4(d), being entirely composed of $\mathrm{Cu} 4 s$ and $3 d_{z^{2}}$ states. The change may favor in-plane superconductivity, as it has been shown that the single-layer cuprate superconductor having the highest $T_{\mathrm{c}}, \mathrm{HgBa}_{2} \mathrm{CuO}_{4}$, has the most $\mathrm{Cu} 4 s$ character in its axial orbital [21]. However, later studies of the role of apical bonding have not all found the same conclusion. One recent study found no correlation between apical bond length and $T_{\mathrm{c}}$ across many different cuprates, and instead found $T_{\mathrm{c}}$ was correlated with the strength of the bond between the $\mathrm{O}(4)$ and the chain $\mathrm{Cu}$ [23].

A second well-known model focuses on out-of-plane transport, treating the $\mathrm{CuO}_{2}$ planes as perfect superconductors and modeling conductivity out of plane as tunneling through a Josephson junction [24]. According to this model, the tunneling barrier between $\mathrm{CuO}_{2}$ planes is the limiting factor, and anything that shortens the planar $\mathrm{Cu}-\mathrm{O}(4)$ bond might raise $T_{\mathrm{c}}$. For example, vacancy-ordered $\mathrm{YBCO}_{6.5}$ showed coherent transport near room temperature on optically exciting a phonon mode specific to the $\mathrm{Cu}-\mathrm{O}(4)$ bond $[25,26]$. Timeresolved diffraction and theoretical calculations indicated that the excited mode increased planar buckling [26], which ought to reduce in-plane transport but improve out-of-plane transport. According to this model, apical $V_{\mathrm{O}}$ is likely to weaken the out-of-plane superconductivity.

\section{CONCLUSION}

In summary, we have reported direct observation of apical $V_{\mathrm{O}}$ in optimally doped YBCO. We find that for low vacancy concentrations, isolated apical and chain $V_{\mathrm{O}}$ has similar formation energy. Apical $V_{\mathrm{O}}$ significantly distort the surrounding lattice and the electronic structure of YBCO and are likely to influence its superconductive properties, although the underlying mechanisms of cuprate superconductivity are not yet understood well enough to predict the exact effect. A strategy to understand the role of the apical vacancies could 
be to apply a stimulus, such as strain, to stabilize apical $V_{\mathrm{O}}$ over chain $V_{\mathrm{O}}$ and monitor the changes in superconducting transport. Likewise, the association between apical vacancies and stacking faults also need to be investigated. It is clear that future efforts to understand the high- $T_{\mathrm{c}}$ superconductivity of YBCO, need to take into account the role of apical $V_{\mathrm{O}}$ on an equal footing as the doping by chain $V_{\mathrm{O}}$.

\section{ACKNOWLEDGMENTS}

This work was partially supported by the National Science Foundation (NSF) Grant No. DMR-1806147 (S.T.H., R.M.). Computations in this work benefited from the use of the Extreme Science and Engineering Discovery Environment (XSEDE), which is supported by NSF Grants No. ACI1053575 and No. ACI-1548562. B.M, J.G., T.P., and X.O acknowledge financial support from the Spanish Ministry of Science Innovation and Universities through the 'Severo Ochoa' Programme for Centres of Excellence in R\&D (SEV-20150496), and the COACHSUPENERGY project (SUMATE RTI2018-095853-B-C21, co-financed by the European Regional Development Fund). They also thank the European
Union for its support under the ULTRASUPERTAPE project (ERC-2014-ADG-669504), and COST Action NANOCOHYBRI (CA16218), and from the Catalan Government under 2017-SGR-1519 and XRE4S. J.G. also acknowledges the Ramon y Cajal program (RYC-2012-11709). STEM imaging and analysis at $200 \mathrm{kV}$ was sponsored by the US Department of Energy (DOE), Office of Science, Basic Energy Sciences, Materials Sciences and Engineering Division, and STEM imaging at $100 \mathrm{kV}$ was conducted at the Center for Nanophase Materials Sciences, which is a DOE Office of Science User Facility (J.-C.I.).

This work has been partially supported by US DOE Grant No. DE-FG02-13ER41967. ORNL is managed by UTBattelle, LLC, under Contract No. DE-AC05-00OR22725 for the US DOE. The US Government retains and the publisher, by accepting the article for publication, acknowledges that the United States Government retains a non-exclusive, paid-up, irrevocable, worldwide license to publish or reproduce the published form of this manuscript, or allow others to do so, for US Government purposes. The Department of Energy will provide public access to these results of federally sponsored research in accordance with the DOE Public Access Plan [27].
[1] R. J. Cava, B. Batlogg, K. M. Rabe, E. A. Rietman, P. K. Gallagher, and L. W. Rupp, Physica C 156, 523 (1988).

[2] E. F. Talantsev, N. M. Strickland, S. C. Wimbush, J. G. Storey, J. L. Tallon, and N. J. Long, Appl. Phys. Lett. 104, 242601 (2014).

[3] G. Deutscher, APL Mater. 2, 096108 (2014).

[4] J. D. Jorgensen, M. A. Beno, D. G. Hinks, L. Soderholm, K. J. Volin, R. L. Hitterman, J. D. Grace, I. K. Schuller, C. U. Segre, K. Zhang, and M. S. Kleefisch, Phys. Rev. B 36, 3608 (1987).

[5] J. Gazquez, R. Guzman, R. Mishra, E. Bartolomé, J. Salafranca, C. Magén, M. Varela, M. Coll, A. Palau, S. M. Valvidares, P. Gargiani, E. Pellegrin, J. Herrero-Martin, S. J. Pennycook, S. T. Pantelides, T. Puig, and X. Obradors, Adv. Sci. 3, 1500295 (2016).

[6] A. Filippetti, G. M. Lopez, M. Mantega, and V. Fiorentini, Phys. Rev. B 78, 233103 (2008).

[7] J. D. Jorgensen, S. Pei, P. Lightfoor, H. Shi, A. P. Paulikas, and B. W. Veal, Physica C 167, 571 (1990).

[8] J. D. Jorgensen, H. Shaked, D. G. Hinks, B. Dabrowski, B. W. Veal, A. P. Paulikas, L. J. Nowicki, G. W. Crabtree, W. K. Kwok, L. H. Nunez, and H. Claus, Physica C 153-155, 578 (1988).

[9] A. P. Shapovalov, M. B. Yu, A. I. Ruban, G. G. Gridneva, V. S. Melnikov, and N. P. Pshentsova, Supercond. Sci. Technol. 5, 283 (1992).

[10] J. C. Cheang Wong, C. Ortega, J. Siejka, I. Trimaille, A. Sacuto, L. M. Mercandalli, and F. Mayca, J. Alloys Compd. 195, 675 (1993).

[11] J. L. MacManus-Driscoll, J. A. Alonso, P. C. Wang, T. H. Geballe, and J. C. Bravman, Physica C 232, 288 (1994).

[12] S. H. Pan, E. W. Hudson, and J. C. Davis, Rev. Sci. Instrum. 70, 1459 (1999).

[13] T. Nishizaki, M. Maki, and N. Kobayashi, J. Phys. Chem. Solids 69, 3014 (2008).

[14] T. Nishizaki, K. Shibata, M. Maki, and N. Kobayashi, J. Low Temp. Phys. 131, 931 (2003).
[15] M. Maki, T. Nishizaki, K. Shibata, and N. Kobayashi, J. Phys. Soc. Jpn. 70, 1877 (2001).

[16] See Supplemental Material at http://link.aps.org/supplemental/ 10.1103/PhysRevMaterials.3.114806 for experimental and computational details, as well as additional STEM-EELS data and DFT results.

[17] R. Liang, D. A. Bonn, and W. N. Hardy, Phys. Rev. B 73, 180505(R) (2006).

[18] R. Ishikawa, E. Okunishi, H. Sawada, Y. Kondo, F. Hosokawa, and E. Abe, Nat Mater 10, 278 (2011).

[19] J. Gazquez, M. Stengel, R. Mishra, M. Scigaj, M. Varela, M. A. Roldan, J. Fontcuberta, F. Sanchez, and G. Herranz, Phys. Rev. Lett. 119, 106102 (2017).

[20] N. Gauquelin, D. G. Hawthorn, G. A. Sawatzky, R. X. Liang, D. A. Bonn, W. N. Hardy, and G. A. Botton, Nat. Commun. 5, 4275 (2014).

[21] E. Pavarini, I. Dasgupta, T. Saha-Dasgupta, O. Jepsen, and O. K. Andersen, Phys. Rev. Lett. 87, 047003 (2001).

[22] Y. Y. Peng, G. Dellea, M. Minola, M. Conni, A. Amorese, D. Di Castro, G. M. De Luca, K. Kummer, M. Salluzzo, X. Sun, X. J. Zhou, G. Balestrino, M. Le Tacon, B. Keimer, L. Braicovich, N. B. Brookes, and G. Ghiringhelli, Nat. Phys. 13, 1201 (2017).

[23] S. Kim, X. Chen, W. Fitzhugh, and X. Li, Phys. Rev. Lett. 121, 157001 (2018).

[24] P. W. Anderson, Science 279, 1196 (1998).

[25] S. Kaiser, C. R. Hunt, D. Nicoletti, W. Hu, I. Gierz, H. Y. Liu, M. Le Tacon, T. Loew, D. Haug, B. Keimer, and A. Cavalleri, Phys. Rev. B 89, 184516 (2014).

[26] R. Mankowsky, A. Subedi, M. Forst, S. O. Mariager, M. Chollet, H. T. Lemke, J. S. Robinson, J. M. Glownia, M. P. Minitti, A. Frano, M. Fechner, N. A. Spaldin, T. Loew, B. Keimer, A. Georges, and A. Cavalleri, Nature (London) 516, 71 (2014).

[27] http://energy.gov/downloads/doe-public-access-plan. 